\title{
Enhanced recovery protocols after pelvic floor reconstructive surgery
}

\section{Dimitrios Zacharakis, Thomas Ntounis, Michail Diakosavvas, Nikolaos Kathopoulis, Christos Kalantzis, Stavros Athanasiou, Themos Grigoriadis}

Urogynecology Unit, 1st Department of Obstetrics and Gynecology, Alexandra Hospital, Faculty of Medicine, National and Kapodistrian University of Athens, Athens, Greece

\section{Corresponding Author}

Dimitrios Zacharakis, PhD, Urogynecology Unit, 1st Department of Obstetrics and Gynecology, Alexandra Hospital, Faculty of Medicine, National and Kapodistrian University of Athens, Athens, Greece, 80 Vasilissis Sofias Avenue, 11528, Athens, Greece. Tel.: +306976 403626, e-mail: dimzac@hotmail.com

\section{Abstract}

Introduction: Enhanced Recovery After Surgery (ERAS) protocols consist of several evidence-based interventions that have been adopted to ameliorate perioperative care. Until this day, no such specific protocols suggested for urogynecology patients, exist. However, there are recent efforts towards the development of such protocols, as it has been shown to have beneficial effects among patients undergoing other gynecological procedures. This review aims to collect and analyze data in the published literature regarding implementation of an enhanced recovery after surgery pathway in a urogynecology population.

Material and Methods: An extensive search of the PubMed/Medline and the Cochrane Database was conducted using the following terms: Enhance Recovery After Surgery combined with pelvic floor reconstructive surgery and urogynecology. Related articles from the latest two decades up to May 2021 were scanned for relevance.

Results: The interventions concerning the implementation of an enhanced recovery pathway after urogynecologic procedures include preoperative, intraoperative, and postoperative recommendations. The main key items in these protocols comprise of patient education, optimization of comorbid conditions, diet, bowel preparation, antibiotic prophylaxis, fluid intake, anesthesiologic agents, early ambulation, postoperative analgesia, thromboprophylaxis and guidance after discharge.

Conclusions: Implementation of an ERAS protocol in urogynecology-specific population has been proven to have a beneficial effect on decreasing hospital stay without increasing the rate of post-operative complications. Nonetheless, the scarcity of evidence-based studies in the literature, regarding the implementation of an ERAS protocol in gynecologic pelvic floor reconstructive surgery is apparent. Hence, further research is required with studies targeted towards urogynecology for safer conclusions to be made.

Key words: Enhanced recovery after surgery, pelvic floor reconstructive surgery, perioperative care, urogynecology 


\section{Introduction}

Enhanced Recovery After Surgery (ERAS) protocols consist of several evidence-based interventions that have been adopted to ameliorate perioperative care. It has been shown, that even though the implementation of the ERAS protocols has optimized the clinical outcome and has reduced the cost of perioperative care, there is a relatively slow adoption and realization of these protocols ${ }^{1}$. Implementation of ERAS protocols require a multidisciplinary complex approach from all health care providers (nursing staff, surgeons, and anesthesiologists), as well as support staff. In some cases, this is found to be particularly challenging, affecting the overall efficacy of a certain protocol ${ }^{2}$.

ERAS protocols have started in 2001 after the so called fast-track protocols in the 1990s, with the first been developed for patients undergoing colorectal surgery ${ }^{3}$. Since and after that time, several protocols have been proposed and introduced for several other types of surgeries, including gynecologicaloncological and pelvic floor surgical procedures ${ }^{4-6}$. Recent efforts have been focused on the development of a urogynecology-specific ERAS protocol' .

Several common actions are found in all ERAS protocols, including preoperative, intraoperative, and postoperative interventions. On a preoperative level such interventions include appropriate screening, nutrition, and patient education. Intraoperatively, factors such as perioperative fluid management, maintenance of temperature homeostasis and opioidsparing analgesia are commonly found in most of the ERAS protocols. Lastly, postoperatively, most ERAS protocols include interventions on early patient mobilization and early oral intake initiation ${ }^{8,9}$.

Although there are no specific ERAS protocols suggested for urogynecology patients from the ERAS society at the moment, there are recent efforts towards the development of such protocols, as it has been shown to have beneficial effects among pa- tients undergoing other gynecological procedures ${ }^{7,10}$. This review aims to collect and analyze data in the published literature regarding implementation of an enhanced recovery after surgery pathway in a urogynecology population.

\section{Materials and Methods}

Using a protocol registered with OSF registries (Registration DOI: 10.17605/OSF.IO/Q5ZBR), we conducted an extensive search of the PubMed/ Medline and the Cochrane Database. The following terms were employed: Enhance Recovery After Surgery combined with pelvic floor reconstructive surgery and urogynecology. Related articles from the latest two decades up to May 2021 were scanned for relevance. We applied no restriction to region, publication type or article language. The reference lists of all eligible published articles were cross-checked, and any additional studies identified were included in the review. Since this study is a review, it did not require Institutional Review Board (IRB) approval.

Our objective is to determine and summarize the interventions of an ERAS protocol targeted for urogynecological patients that would result in optimization of their surgical experience and higher patient satisfaction (Table 1).

\section{Results}

\section{Preoperative interventions}

Patient counseling and education

ERAS protocols on pelvic floor surgery including those for gynecologic-oncology and colorectal surgery commend that patient counseling and education, prior to admission are essential elements ${ }^{11,12}$. Medical history and comorbidities assessment are the core stone of preoperative optimization. Additional assessment of exercise tolerance and lifestyle factors are directly related to enhanced peri- and postoperative outcomes. 
Table1. ERAS Protocols in Urogynecology

\begin{tabular}{|c|c|c|}
\hline \multirow{2}{*}{$\begin{array}{l}\text { ERAS ITEM } \\
\text { Preoperative } \\
\text { Interventions }\end{array}$} & \multirow[b]{2}{*}{ Counseling and Education } & RECOMMENDATION \\
\hline & & $\begin{array}{l}\text { Verbal counselling } \\
\text { Handout delivery detailing the procedure and describing recovery process }\end{array}$ \\
\hline \multirow{9}{*}{$\begin{array}{l}\text { Intraoperative } \\
\text { Interventions }\end{array}$} & Comorbid Conditions & $\begin{array}{l}\text { Smoking cessation } \\
\text { Control of Diabetes Mellitus } \\
\text { Patient status optimization (anemia, hypertension) }\end{array}$ \\
\hline & Diet & $\begin{array}{l}\text { Carbohydrate loading } \\
\text { Avoidance of preoperative fasting } \\
\text { Clear fluids up to } 2 \text { hours before surgery }\end{array}$ \\
\hline & Bowel Preparation & Unnecessary \\
\hline & $\begin{array}{l}\text { Nausea and Vomiting } \\
\text { Prevention }\end{array}$ & $\begin{array}{l}\text { Use of more than two antiemetic agents } \\
\text { Gabapentine and Pregabaline }\end{array}$ \\
\hline & Temperature Homeostasis & Active warming of patients during vaginal surgery \\
\hline & Antibiotic Prophylaxis & $\begin{array}{l}\text { Additional data needed to reach into safe conclusions for pelvic floor } \\
\text { reconstructive surgeries }\end{array}$ \\
\hline & Tubes and Drains & $\begin{array}{l}\text { Removal of transurethral catheters as soon as possible } \\
\text { Avoidance of routine use of peritoneal drain catheters }\end{array}$ \\
\hline & Anaesthesia & $\begin{array}{l}\text { Short-acting administration of anesthetics } \\
\text { Supplementary perioperative and postoperative analgesia } \\
\text { Use of lidocaine, regional block or epidural anesthesia }\end{array}$ \\
\hline & Fluid Intake & Perioperative euvolemia and perioperative fluid optimization \\
\hline \multirow{6}{*}{$\begin{array}{l}\text { Postoperative } \\
\text { Interventions }\end{array}$} & Ambulation & Early ambulation \\
\hline & Postoperative Analgesia & $\begin{array}{l}\text { Opioid-sparing analgesia } \\
\text { Multimodal analgesia }\end{array}$ \\
\hline & Oral Diet & Early oral feeding in the first 24 hours after surgery \\
\hline & Prevention of Ileus & $\begin{array}{l}\text { Conflicting evidence whether gum chewing, or the use of laxatives may have a } \\
\text { beneficial effect after surgery }\end{array}$ \\
\hline & Thromboprophylaxis & $\begin{array}{l}\text { Pneumatic compression devices and/or compression stockings } \\
\text { Low molecular weight heparin }\end{array}$ \\
\hline & Discharge and follow-up & $\begin{array}{l}\text { Specific discharge criteria } \\
\text { Postoperative counseling and education } \\
\text { Postoperative questionnaire }\end{array}$ \\
\hline
\end{tabular}

Preoperative education before a surgical procedure has been associated with higher preparedness and satisfaction, during and after surgery. The means to educate and prepare patients include not only verbal counselling, but also handout delivery detailing the procedure and describing recovery process. Moreover, there is evidence that preoperative education aids on the psychological status of patients and may have a beneficial attribute on the length of post-operative stay, in the management of pain and the whole recovery process ${ }^{11-13}$.

\section{Optimization of comorbid conditions}

Patient status optimization of comorbid conditions, such as diabetes mellitus, anemia, and hypertension, were also associated with enhanced recovery outcomes, while early recovery after surgery has been reported with psychological and physical interven- 
tions, such as exercise and nutritional supplementation prior to surgery ${ }^{14,15}$.

In detailed, preoperative interventions on comorbid conditions such as metabolic syndrome, diabetes and smoking were proven to have a beneficial effect on patients undergoing a surgical procedure. Smoking is well known that is associated with increased postoperative complication risk ${ }^{16,17}$. A recent research has shown that smoking cessation even for a short term (three to four weeks) prior to surgery has reduced postoperative complication rates, as it reduces both the risk of wound healing and respiratory complications ${ }^{18}$. Diabetes mellitus is also known to be associated with postoperative complications, such as incision site infection, therefore preoperative management, and better control of glycated haemoglobin (HbA1c) levels may reduce the risk of peri- and post-operative complications ${ }^{19}$.

Diet

Another common intervention seen in most ERAS protocols is the so called "nil per mouth (NPO) after midnight" theory challenge. Modern approach in ERAS protocols suggest, carbohydrate loading and avoidance of preoperative fasting. Thirst reduction by administration of clear fluids up to two hours before surgery has been shown to be beneficial in overall patient status, relatively safe and it was associated with lower gastric volumes ${ }^{20}$. Furthermore, some ERAS protocols suggest administration of carbohydrate rich fluids even as early as two hours before surgery ${ }^{21}$. Patients by this way, postoperatively show higher protein and glucose metabolism, in contrast to patients in absence state (traditional NPO approach). It has shown to be safe even in well-controlled diabetic patients if administered three hours before surgery and does not influence gastric emptying ${ }^{22}$.

\section{Bowel preparation}

Several ERAS guidelines support avoidance of mechanical bowel preparation or the use of colorectal enemas, as it does not provide any benefit and it is not associated with increased morbidity ${ }^{23,24}$. It has been proven that mechanical bowel preparation may cause electrolyte disturbances and has a negative effect on preoperative patient homeostasis, as it may cause dehydration ultimately affecting the perioperative and postoperative outcome ${ }^{24}$. Thus, according to recent evidence, any type of preoperative bowel preparation, mechanical or with oral antibiotics, should be omitted prior to urogynecological surgical procedures $^{25-28}$.

\section{Intraoperative interventions}

Nausea and vomiting prevention

Intraoperative nausea and vomiting prevention are common interventions seen in ERAS protocols. Multimodal approach with the use of more than two antiemetic agents has been proven to be more effective than a single drug therapy. Several drugs used as monotherapy including droperidol, ondansetron and dexamethasone where more effective than nitrogen and propofol ${ }^{29}$. Additional factors such as gabapentine and pregabaline, were also seen to be effective against nausea and vomiting ${ }^{30}$.

\section{Maintenance of temperature homeostasis}

ERAS protocols pay also particular importance in the maintenance of patient's temperature homeostasis. Several ERAS protocols suggest that active warming for patients undergoing pelvic floor and gynecologic-oncologic surgery is strongly recommended. There is a strong evidence that active warming has a beneficial effect in surgical wound healing and on the prevention of postoperative surgical incision site infection ${ }^{31}$.

\section{Antibiotic prophylaxis}

Prophylactic antibiotic administration is abundant on surgical practice. A 2014 review supports that the percentage of postoperative infection risk was reduced from $39 \%$ to $13 \%$ after administration of prophylactic 
antibiotics in colorectal surgery, with the most effective administration pattern being the combination of both intravenous and oral antibiotics ${ }^{32}$. However, additional data may be necessary to reach into safe conclusions for pelvic floor reconstructive surgeries.

\section{Tubes and drains}

In most revised ERAS protocols, the predominant idea is the avoidance of peritoneal drains, urinary catheters, and nasogastric tubes when it is relatively possible. There is lack of evidence whether the use of peritoneal drain catheter might have a beneficial effect on patients undergoing pelvic floor surgery and thus their routine use should be avoided. A meta-analysis supports that the use of a nasogastric tube should be rather selective than routine, as its use is associated with increased risk of pulmonary complications and late bowel function mobilization ${ }^{33}$. On patients undergoing pelvic floor reconstructive surgery with relatively low risk of urinary retention ERAS protocols suggest that transurethral catheters should be removed as early as possible.

\section{Anesthetic approach}

Short-acting administration of anesthetics and the use of a standardized anesthetic protocol has been found to be associated with early bowel mobilization after surgery and reduced length of hospital stay. Additionally, elimination of stress response, rapid awakening and minimal residual effects are the main reasons for the use of short-acting general anesthetics ${ }^{34}$.

The standardized anesthetic protocols found in all revised ERAS protocols, include as supplementary perioperative and postoperative analgesia, the use of lidocaine, regional block, or epidural anesthesia ${ }^{4}$. Local anesthesia has also been utilized as an alternative anesthetic method for pelvic floor reconstructive surgery $^{35}$. Local anesthesia has known advantages over both regional and general anesthesia techniques such as minimal interference with homeostasis, a lower risk of postoperative nausea, vomiting, delirium and cognitive dysfunction, a lower need for postoperative analgesia and an earlier fulfillment of discharge criteria ${ }^{35}$.

\section{Fluid management}

An essential part of all ERAS protocols regarding the standardization of the anesthetics protocol is the perioperative management of fluids. All ERAS protocols are directed towards perioperative euvolemia and perioperative fluid optimization. Dehydration or overhydration should be avoided in pelvic floor surgery. All revised ERAS guidelines impose the use of doppler intraoperative fluid optimization, which is related with overall decreased risk of wound infection and postoperative ileus, as well as with decreased incidence of nausea and vomiting ${ }^{36}$.

\section{Postoperative interventions}

Early ambulation

Early ambulation is an essential part of all ERAS protocols. Although there is lack of evidence connecting improved clinical outcomes in patients undergoing pelvic floor reconstructive surgery, there are plenty of trials in abdominal surgery that connect early mobilization to early bowel function mobilization and improved clinical outcomes with reduced length of hospitalization ${ }^{37}$. Moreover, according to several trials the negative effects of bed rest such as insulin resistance, pulmonary dysfunction and musculature loss are well known ${ }^{38}$.

\section{Multimodal post-operative analgesia}

Opioid-sparing analgesia is another innovation included in all revised ERAS protocols. According to the literature, multimodal analgesia in pelvic floor reconstructive surgery was found to have an equal effect on pain reduction without requiring the use of opioids. Moreover, opioid free analgesia spares the use of other pharmacological agents, such as antiemetics to counteract opioid's side effects ${ }^{39}$. 


\section{Early oral diet}

In all recommended revised ERAS protocols early, oral feeding is strongly recommended, as it has been proven that there is lower incidence of complications. This is particularly significant in urogynecology patients and therefore it is strongly recommended. Early oral feeding is defined as oral intake of fluids and food earlier than 24 hours postoperatively ${ }^{40}$.

\section{Prevention of ileus}

A serious postoperative complication that needs to be considered is ileus. All ERAS guidelines include various method to avoid such a serious complication. There is conflicting evidence whether gum chewing, or the use of laxatives may have a beneficial effect after surgery, especially in urogynecological patients 4 . Moreover, a recent study suggests that post-operative ileus, as well as opioid induced dysfunction of the bowel, can be effectively treated with the use of peripherally acting opioid-receptor antagonists such as alvimopam ${ }^{41}$.

\section{Thromboprophylaxis}

Another field of particular importance in all ERAS guidelines, is the venous thromboembolism prophylaxis. A recent review found that when mechanical prophylaxis is combined with pharmacological prophylaxis, may even more reduce the incidence of deep vein thrombosis from 4.1 to $2.9 \%{ }^{42}$. Mechanical thromboprophylaxis in the recommended ERAS protocols include pneumatic compression devices and/or compression stockings, while the pharmacological agent used, is low molecular weight heparin which is administered for 28 days $^{43}$.

\section{Patient discharge and follow-up}

ERAS guidelines do not propose a specific followup and discharge protocol for patients undergoing pelvic floor surgery, although they may include some interventions regarding this topic. A recent research proposing the implementation of an urogynecology -specific protocol included specific discharge criteria such as, low pain scores (less than five), uncomplicated voiding, ambulation and ingestions of foods and fluids, as well as delirium absence ${ }^{7}$. Another study suggests the use of additional criteria such as specific laboratory results (including full blood count and c-reactive protein levels) and findings after clinical evaluation (including vital signs, abdominal physical examination findings and overall patient well-being evaluation $)^{44}$.

Finally, it was shown that there was a significant higher major complication rate on patients with pain score more than four, unwilling patients to be discharged and patients with white blood count over $14.000 \mathrm{k} / \mathrm{ml}$. Another intervention that may be applicable on urogynecology patients would be post-operative counseling and education. This observation is based on another research study performed upon patients undergoing ileostomy procedures, on which their knowledge regarding their familiarization with the management of appliances was tested, finally showing that active participation showed a significant rate reduction in readmissions ${ }^{45}$. Another significant finding was, that detection of postoperative complications reached approximately $80 \%$ when a questionnaire after two months and a two-week follow-up was imposed, in contrast to a $20 \%$ detection rate shown when solely clinical audit was performed, on patients undergoing various surgeries ${ }^{46}$.

\section{Discussion}

The ERAS concept has been recently developed for many gynecological surgical procedures and for urogynecology patients 7 . ERAS protocols interventions have been shown to be a feasible alternative to standard practice in women undergoing pelvic floor reconstructive surgery offering an enhanced recovery and high patient satisfaction rate. This is particular important for geriatric patients or patients 
with comorbidities requiring a reduced hospital stay.

In a retrospective research analysis, a total of 258 women undergoing a major pelvic organ prolapse surgery were studied. After the implementation of urogynecology-specific ERAS pathway, it was shown that patients had higher probability of same day discharge and decreased length of admission ${ }^{7}$. Additionally, patients reported preparedness for surgery by $89.6 \%$, excellent or very good pain management by $86.7 \%$ and an overall excellent surgical experience by $93.7 \%$. Another important element was that during postoperative recovery about $90 \%$ did not show any signs of nausea. The authors accredit this to a holistic adoption of all the ERAS components from all the implicated entities and hospital staff.

\section{Conclusions}

Implementation of an ERAS protocol in urogynecology-specific targeted population has been proven to have a beneficial effect on decreasing hospital stay without concomitantly increasing the rate of post-operative complications.

Nonetheless, the scarcity of evidence-based studies in the literature, regarding the implementation of an ERAS protocol in gynecologic pelvic floor reconstructive surgery is apparent. Hence, further research is required with studies targeted towards urogynecology for safer conclusions to be made.

\section{Conflict of Interest}

All authors declare no conflict of interest.

\section{References}

1. Ljungqvist O, Scott M, Fearon KC (2017) Enhanced Recovery After Surgery. JAMA Surg 152:292. https://doi.org/10.1001/jamasurg.2016.4952

2. Ljungqvist $O$ (2014) ERAS-Enhanced Recovery After Surgery. J Parenter Enter Nutr 38:559-566. https://doi.org/10.1177/0148607114523451

3. Lassen K (2009) Consensus Review of Opti- mal Perioperative Care in Colorectal Surgery. Arch Surg 144:961. https://doi.org/10.1001/ archsurg.2009.170

4. Nelson G, Bakkum-Gamez J, Kalogera E, et al (2019) Guidelines for perioperative care in gynecologic/oncology: Enhanced Recovery After Surgery (ERAS) Society recommendations - 2019 update. Int J Gynecol Cancer 29:651-668. https:// doi.org/10.1136/ijgc-2019-000356

5. Gustafsson UO, Scott MJ, Hubner M, et al (2019) Guidelines for Perioperative Care in Elective Colorectal Surgery: Enhanced Recovery After Surgery (ERAS $®$ ) Society Recommendations: 2018. World J Surg 43:659-695. https://doi. org/10.1007/s00268-018-4844-y

6. Nygren J, Thacker J, Carli F, et al (2012) Guidelines for perioperative care in elective rectal/pelvic surgery: Enhanced Recovery After Surgery (ERAS®) Society recommendations. Clin Nutr 31:801-816. https://doi.org/10.1016/j.clnu.2012.08.012

7. Carter-Brooks CM, Du AL, Ruppert KM, et al (2018) Implementation of a urogynecology-specific enhanced recovery after surgery (ERAS) pathway. Am J Obstet Gynecol 219:495.e1-495. e10. https://doi.org/10.1016/j.ajog.2018.06.009

8. Barber EL, Van Le L (2015) Enhanced Recovery Pathways in Gynecology and Gynecologic Oncology. Obstet Gynecol Surv 70:780-792. https://doi. org/10.1097/OGX.0000000000000259

9. Cohen R, Gooberman-Hill R (2019) Staff experiences of enhanced recovery after surgery: systematic review of qualitative studies. BMJ Open 9:e022259. https://doi.org/10.1136/ bmjopen-2018-022259

10. Kalogera E, Bakkum-Gamez JN, Jankowski CJ, et al (2013) Enhanced Recovery in Gynecologic Surgery. Obstet Gynecol 122:319-328. https://doi. org/10.1097/AOG.0b013e31829aa780

11. Yi HC, Ibrahim Z, Abu Zaid Z, et al (2020) Impact of Enhanced Recovery after Surgery with Preop- 
erative Whey Protein-Infused Carbohydrate Loading and Postoperative Early Oral Feeding among Surgical Gynecologic Cancer Patients: An OpenLabelled Randomized Controlled Trial. Nutrients 12:264. https://doi.org/10.3390/nu12010264

12. Powell R, Scott NW, Manyande A, et al (2016) Psychological preparation and postoperative outcomes for adults undergoing surgery under general anaesthesia. Cochrane Database Syst Rev 2016:CD008646. https://doi. org/10.1002/14651858.CD008646.pub2

13. Zambouri A (2007) Preoperative evaluation and preparation for anesthesia and surgery. Hippokratia 11:13-21

14. Jensen BT, Lauridsen S V., Jensen JB (2018) Prehabilitation for major abdominal urologic oncology surgery. Curr Opin Urol 28:243-250. https://doi. org/10.1097/MOU.0000000000000487

15. Miralpeix E, Mancebo G, Gayete S, et al (2019) Role and impact of multimodal prehabilitation for gynecologic oncology patients in an Enhanced Recovery After Surgery (ERAS) program. Int J Gynecol Cancer 29:1235-1243. https://doi. org/10.1136/ijgc-2019-000597

16. Thomsen T, Tønnesen H, Møller AM (2009) Effect of preoperative smoking cessation interventions on postoperative complications and smoking cessation. Br J Surg 96:451-461. https://doi. org/10.1002/bjs.6591

17. Wong J, Lam DP, Abrishami A, et al (2012) Shortterm preoperative smoking cessation and postoperative complications: a systematic review and meta-analysis. Can J Anesth Can d'anesthésie 59:268-279. https://doi.org/10.1007/ s12630-011-9652-x

18. Gustafsson UO, Thorell A, Soop M, et al (2009) Haemoglobin A1c as a predictor of postoperative hyperglycaemia and complications after major colorectal surgery. Br J Surg 96:1358-1364. https://doi.org/10.1002/bjs.6724
19. Brady MC, Kinn S, Stuart P, Ness V (2003) Preoperative fasting for adults to prevent perioperative complications. Cochrane Database Syst Rev 2003:CD004423. https://doi. org/10.1002/14651858.CD004423

20. Nygren J (2006) The metabolic effects of fasting and surgery. Best Pract Res Clin Anaesthesiol 20:429-438. https://doi.org/10.1016/j. bpa.2006.02.004

21. Bilku D, Dennison A, Hall T, et al (2014) Role of preoperative carbohydrate loading: a systematic review. Ann R Coll Surg Engl 96:15-22. https:// doi.org/10.1308/003588414X13824511650614

22. Arnold A, Aitchison LP, Abbott J (2015) Preoperative Mechanical Bowel Preparation for Abdominal, Laparoscopic, and Vaginal Surgery: A Systematic Review. J Minim Invasive Gynecol 22:737-752. https://doi.org/10.1016/j.jmig.2015.04.003

23. Güenaga KF, Matos $\mathrm{D}$, Wille-Jørgensen $\mathrm{P}$ (2011) Mechanical bowel preparation for elective colorectal surgery. Cochrane Database Syst Rev 2011:CD001544. https://doi. org/10.1002/14651858.CD001544.pub4

24. Diakosavvas M, Thomakos N, Haidopoulos D, et al (2020) Controversies in preoperative bowel preparation in gynecologic and gynecologic oncology surgery: a review of the literature. Arch Gynecol Obstet. https://doi.org/10.1007/ s00404-020-05704-1

25. Diakosavvas M, Thomakos N, Psarris A, et al (2020) Preoperative Bowel Preparation in Minimally Invasive and Vaginal Gynecologic Surgery. Sci World J 2020:1-7. https://doi. org/10.1155/2020/8546037

26. Adelowo AO, Hacker MR, Modest AM, et al (2017) The Use of Mechanical Bowel Preparation in Pelvic Reconstructive Surgery: A Randomized Controlled Trial. Female Pelvic Med Reconstr Surg 23:1-7. https://doi.org/10.1097/ SPV.0000000000000346 
27. Deng H, Liu YY, Tan C, et al (2019) [A randomized single blind comparison of conventional bowel preparation and unplanned preoperative preparation for pelvic organ prolapse]. Zhonghua Fu Chan Ke Za Zhi 54:97-102. https://doi. org/10.3760/cma.j.issn.0529-567x.2019.02.005

28. Ballard AC, Parker-Autry CY, Markland AD, et al (2014) Bowel Preparation Before Vaginal Prolapse Surgery. Obstet Gynecol 123:232-238. https:// doi.org/10.1097/AOG.0000000000000081

29. Grant MC, Betz M, Hulse M, et al (2016) The Effect of Preoperative Pregabalin on Postoperative Nausea and Vomiting. Anesth Analg 123:1100-1107. https://doi.org/10.1213/ ANE.0000000000001404

30. Kim KM, Huh J, Lee SK, et al (2017) Combination of gabapentin and ramosetron for the prevention of postoperative nausea and vomiting after gynecologic laparoscopic surgery: a prospective randomized comparative study. BMC Anesthesiol 17:65. https://doi.org/10.1186/s12871-017-0357-8

31. Madrid E, Urrútia G, Roqué i Figuls M, et al (2016) Active body surface warming systems for preventing complications caused by inadvertent perioperative hypothermia in adults. Cochrane Database Syst Rev 2016:CD009016. https://doi. org/10.1002/14651858.CD009016.pub2

32. Liu Z, Dumville JC, Norman G, et al (2018) Intraoperative interventions for preventing surgical site infection: an overview of Cochrane Reviews. Cochrane Database Syst Rev 2018:CD012653. https://doi.org/10.1002/14651858.CD012653. pub2

33. Verma R, Nelson RL (2007) Prophylactic nasogastric decompression after abdominal surgery. Cochrane Database Syst Rev 2007:CD004929. https://doi.org/10.1002/14651858.CD004929. pub3

34. Marana E, Colicci S, Meo F, et al (2010) Neuroendocrine stress response in gynecological laparos- copy: TIVA with propofol versus sevoflurane anesthesia. J Clin Anesth 22:250-255. https://doi. org/10.1016/j.jclinane.2009.07.011

35. Athanasiou S, Zacharakis D, Grigoriadis T, et al (2020) Vaginal hysterectomy with anterior and posterior repair for pelvic organ prolapse under local anesthesia: results of a pilot study. Int Urogynecol J 31:2109-2116. https://doi.org/10.1007/ s00192-020-04326-0

36. Webb C, Day R, Velazco CS, et al (2020) Implementation of an Enhanced Recovery After Surgery (ERAS) Program is Associated with Improved Outcomes in Patients Undergoing Cytoreductive Surgery and Hyperthermic Intraperitoneal Chemotherapy. Ann Surg Oncol 27:303-312. https:// doi.org/10.1245/s10434-019-07900-z

37. Castelino T, Fiore JF, Niculiseanu P, et al (2016) The effect of early mobilization protocols on postoperative outcomes following abdominal and thoracic surgery: A systematic review. Surgery 159:991-1003. https://doi.org/10.1016/j. surg.2015.11.029

38. Brower RG (2009) Consequences of bed rest. Crit Care Med 37:S422-S428. https://doi. org/10.1097/CCM.0b013e3181b6e30a

39. Reagan KML, O'Sullivan DM, Gannon R, Steinberg AC (2017) Decreasing postoperative narcotics in reconstructive pelvic surgery: a randomized controlled trial. Am J Obstet Gynecol 217:325.e1-325. e10. https://doi.org/10.1016/j.ajog.2017.05.041

40. Herbert G, Perry R, Andersen HK, et al (2018) Early enteral nutrition within 24 hours of lower gastrointestinal surgery versus later commencement for length of hospital stay and postoperative complications. Cochrane Database Syst Rev 2018:CD004080. https://doi. org/10.1002/14651858.CD004080.pub3

41. Schwenk ES, Grant AE, Torjman MC, et al (2017) The Efficacy of Peripheral Opioid Antagonists in Opioid-Induced Constipation and Postoperative 
Ileus. Reg Anesth Pain Med 42:767-777. https:// doi.org/10.1097/AAP.0000000000000671

42. Kakkos SK, Caprini JA, Geroulakos G, et al (2016) Combined intermittent pneumatic leg compression and pharmacological prophylaxis for prevention of venous thromboembolism. Cochrane Database Syst Rev 2016:CD005258. https://doi. org/10.1002/14651858.CD005258.pub3

43. Felder S, Rasmussen MS, King R, et al (2019) Prolonged thromboprophylaxis with low molecular weight heparin for abdominal or pelvic surgery. Cochrane Database Syst Rev 2019:CD004318. https://doi.org/10.1002/14651858.CD004318. pub4

44. Mannaerts GHH, van Mil SR, Stepaniak PS, et al (2016) Results of Implementing an Enhanced Recovery After Bariatric Surgery (ERABS) Protocol. Obes Surg 26:303-312. https://doi.org/10.1007/ s11695-015-1742-3

45. Hardiman KM, Reames CD, McLeod MC, Regenbogen SE (2016) Patient autonomy-centered selfcare checklist reduces hospital readmissions after ileostomy creation. Surgery 160:1302-1308. https://doi.org/10.1016/j.surg.2016.05.007

46. Woodfield J, Deo P, Davidson A, et al (2019) Patient reporting of complications after surgery: what impact does documenting postoperative problems from the perspective of the patient using telephone interview and postal questionnaires have on the identification of complications after surgery? BMJ Open 9:e028561. https://doi. org/10.1136/bmjopen-2018-028561

Received 17-09-21

Revised 24-09-21

Accepted 30-09-21 